\title{
Some Challenges in Using Computer-Aided Translation Tools to Facilitate Second Language Fluency in Education
}

\author{
Dianne Excell \\ Wrexham Glyndŵrr University, UK \\ dianne excell@hotmail.com
}

Received: 6 ${ }^{\text {th }}$ December 2018; Accepted: $3^{\text {rd }}$ February 2019; Published: $1^{\text {st }}$ April 2019

\begin{abstract}
At every level of education, from early years to postgraduate, students can arrive in the UK (or US) classroom with little or no English language ability. It is impossible for teachers to have knowledge of every first language that students bring into the classroom and thus they may rely on a computer-aided translation tool so that their students can have access to the English needed in all subjects across the curriculum. At the same time, students need to move from basic English skills in listening, speaking, reading and writing towards the subtleties of academic English which they must achieve in order to reach the UK government's required standards in English at every level of assessment. Using her extensive knowledge of the challenges that students learning English as an Additional Language (EAL) and their teachers face in the classroom situation and the English needed in assessments, the author examines possible benefits of computer-aided translation tools such as Google Translate and Talking Pen and outlines some of the problems and drawbacks with such tools that create barriers to acquiring full academic fluency, thus posing challenges to future translation tool developers.
\end{abstract}

Keywords: Computer Aided Translation; eLearning; e-translation Tools; English as an Additional Language $(E A L)$

\section{Introduction}

In today's global environment, there are many reasons for the movement of people from one country to another, for example as economic migrants, refugees, asylum seekers, university students or for employment. Often they bring children with them. If these children are of school age, by law, in the United Kingdom (and USA) they are expected to attend an age-appropriate school where they will be taught in English. The languages that these children speak (L1) are usually different from English. In January 2015 [1] there were over 1.5 million pupils in English schools who spoke approximately 320 different languages. In one school alone in Leeds, Yorkshire, 81 different languages are spoken. Understandably, this creates a challenge for teaching and learning when students have to learn the English language at the same time as they are being taught the contents of 
each subject in the curriculum. Computer-aided translation tools are one method of differentiation used to allow students learning English as an additional language (EAL) to access the language used in the curriculum. But are the translation tools available sufficiently effective to ensure that all students learning EAL will have academic success at all levels of assessment? Whilst investigating the benefits and drawbacks of some of the computer-aided translation tools available, this paper will consider the challenges faced by mainstream teachers and the students learning EAL in order to participate in lessons and achieve success in assessments, before making suggestions for computer aided translation tool developers to consider in the future.

\section{Development of computer-based translation tools}

Computer-aided translation is not a new phenomenon. After World War 2, there was a great need for human translators so that scientists could 'keep abreast of Russian scientific publications' [2]. An American scientist and government administrator, Weaver, who had observed the success of British cryptographers in breaking the German Enigma Code realised that machine translation might be possible to solve the problem.

In the early evolutionary period of direct dictation systems, such as IBM ViaVoice, it was noted that recognition of spoken words could be greatly improved by taking context into account. ViaVoice did this by considering "trigrams", meaning sequences of three word sounds: the word being identified, the one before it, and the one after it. This greatly reduced the ambiguity, compared with systems that simply tried to recognise individual words in isolation. Similar issues arise in the removal of ambiguity in translation systems and, with the increasing power of computers, the theory behind the principle has been extended to the concept of " $n$-grams", meaning the use of (n-1) words surrounding the word under consideration in order to try to refine ambiguity out of the identification of the meaning [3].

In the 1980s a change of mindset occurred, between using the expertise of professional translators to develop automatic translation tools and the brute force method of searching large numbers of parallel dual language texts and learning from them. As Lewis-Kraus explains: "The scientific community largely came to accept this view: Machine translation required the help of trained linguists, who would derive increasingly abstract grammatical rules to distil natural languages down to the sets of formal symbols that machines could manipulate" [2].

This paradigm prevailed until 1988, a turning point for modern machine translation, when a team of IBM's speech-recognition researchers presented a new approach. .... "Our approach," they wrote, "eschews the use of an intermediate mechanism (language) that would encode the 'meaning' of the source text." All you had to do was load reams of parallel text through a machine and compute the statistical likelihood of matches across languages.' [2] The problem of semantic ambiguity, which human translators are concerned with, has also been lessened by using a probabilistic model in which the computer examines a few words nearby to gain the correct meaning [3]. In other words human translators would no longer be needed. This statistical method is used by Google Translate and Skype Translator. Google Translate offers 90 different languages and is "probably the most widely used translation tool, used more than a billion times a day worldwide by more than 500 million people a month" [2]. These tools differ from stand-alone direct dictation systems by relying on a relatively wideband Internet link to a powerful central server. This has the advantage that the "knowledge" of the server can be updated in near-real-time, but has the disadvantage of the necessity of the Internet connection.

In 2017, Monroe [4] reported that Google had begun to replace its 'phrase-based system...with some popular language pairs' and the use of neural networks. In 2016 this was described as "essentially as good as human translation, at least for European languages" but, although the improvement for translation is significant, Mair (in [4]) still maintains that "there are still some things they will never get right" between Chinese and English.

Dorren [5] in 2019 examined Google's new "Interpreter Mode" for the Google Assistant which can translate more than 27 languages in real time and stated a belief that in future translation software a "Babel chip" will be able to translate any language in real time. However, he 
acknowledges that it does not seem possible that a Babel chip will be ready in the near future and, even when developed, "worldwide adoption would take even longer."

Research by Kapitanov et al in 2019 [6] proposed "a method of eliminating the polysemy of words based on the clustering of word contexts" in order to improve the quality of text translation into natural language. They reported that, by using this method, it was possible to "improve the algorithms of automatic classification of text documents in natural language" by $1.8 \%$, which amounted to $96.1 \%$ overall. However, this is only a start and they have plans to continue their research into natural language processing in the future: there are still many more challenges, as can be seen in the next section.

\section{Challenges for teachers of students learning EAL}

At every level of education, from early years to postgraduate, students can arrive in the UK (or US) classroom with little or no English language ability at any time in the academic year. Teachers teach their curriculum subject in English and often experience anxiety when they are told that a new arrival will be in their class because they feel they are specialist teachers in their own subject but not teachers of English itself. Some teachers may have knowledge of common community languages spoken in the area around the school but it is impossible for teachers to have knowledge of every first language that students bring into the classroom. Even in schools where there are many students learning EAL, when a student whose first language (L1) is more unusual, teachers wonder how they will fulfil their statutory obligations to ensure the student can access the curriculum and its assessments. For example in Bradford, Yorkshire (UK), historically, most schools have students whose L1 is one of the South Asian languages such as Urdu, Punjabi, Hindi, Bengali, Pushto, Gujarati, Hindko... Many Bradford teachers speak one or more of these languages but, in recent years, there have also been many students arriving from Eastern Europe speaking very different languages such as Polish, Czech, Roma and Turkish that no staff member can speak. Students can come from anywhere in the world and it is not uncommon for students to speak several languages, for example Urdu, Japanese and Arabic. In schools with no history of students learning EAL the challenge to teachers can be even greater. Sometimes there will be in-class support from a teaching assistant but, if computers are available, teachers may provide students with a list of key words and phrases to be translated using a computer-aided translation tool, in the expectation that the students will be able to understand the content of the lesson.

Students learning EAL arrive in the classroom with different levels of ability in both their L1 and English but all have to move from basic English skills in listening, speaking, reading and writing towards the subtleties of academic English which they must achieve in order to reach the UK government's required standards in English at every level of assessment in every subject. So every teacher must now be a teacher of English, whatever subject they teach.

\section{Challenges for students learning EAL in the classroom situation}

Students learning EAL arrive into a classroom where most or all of the other students are native speakers and it can be a daunting experience - especially if they have already suffered the trauma of fleeing their home country or if they have never been to school before. The new arrivals have to build up relationships with both teachers and other students; they often lack confidence and self esteem and therefore sometimes go through a silent period. Learning a new language is not an instant skill: the renowned Canadian professor of language development and literacy, Cummins [7], believes that it can take 2-3 years for a student learning EAL to acquire the basic interpersonal communication skills (BICs) such as peer to peer conversation and classroom language and 3-7 years to achieve the cognitive academic language proficiency (CALP) of a native speaker. Other researchers believe that it can take as much as 7-10 years depending on students' circumstances and linguistic ability in L1. The acquisition of any language involves the four skills of listening, speaking, reading and writing, each of which brings its own challenges. Here the author shares some of the challenges she has observed in her teaching: 


\subsection{Listening}

An EAL learner may mishear much of the spoken content of a lesson delivered at the normal pace, which is often too quick for non-native speakers. Words may appear to run into one another if the teacher does not speak clearly, for example: words such as "have to" may sound like "after"; similar words such as "affect", "effect" and "infect" can be difficult to decipher; abbreviated words such as "could've" may be heard as "could of". Whole words may be omitted and replaced by gestures or facial expressions and word endings such as " $\mathrm{t}$ " in "didn' $\mathrm{t}$ " or beginnings such a " $\mathrm{h}$ " in "house" may be omitted in "lazy" speech. Speech may not be grammatically correct, for example, verb tenses can be mixed up, "is" can be used instead of "are" and past tense endings are heard differently: "id" in "wanted", " $\mathrm{t}$ " in "danced" " $\mathrm{d}$ " in "treated". Additionally, teachers may speak in different regional accents such as Scottish, Birmingham, Essex or Yorkshire, or even foreign accents, adding to the confusion.

\subsection{Speaking}

It is essential that students hear good examples of spoken English if they are to speak with the correct pronunciation. Imperfect pronunciation may occur for example

- if emphasis is put on the wrong syllable in a word, for example in the noun "present" emphasis is placed on the first syllable, whereas in the verb "present" emphasis is on the second - and many words have more than two syllables;

- with vowels a, e, i, o, u - especially with some regional accents where a vowel is lengthened as in "last" sounding like "larst", and "but" becomes "bat".

Examples of confusion in pronunciation when speaking to read aloud are:

- vowels where " $\mathrm{e}$ " is added at the end of a word to lengthen the internal vowel e.g. "mad" is a short vowel sound but "made" is long;

- homonyms (words that are written the same but pronounced differently in different word classes) "live" as a verb has a short vowel, whereas as the adjective "live" it is long; "read" in the present verb tense is a long vowel but "read" in the past tense is short;

- silent letters such as " 1 " in "calm" and " $\mathrm{b}$ " in "climbing" are spoken, thus creating comprehension difficulties;

- names in another language - for example "Simon" has a long " $\mathrm{i}$ " but it looks as though it should be short; "Leicestershire", "Brighouse"...

- letter combinations in words such as "dge" as in "hedge", "ph" as in "photograph", "au" as in "gauge" (pronounced "gayge") could alternatively sound like "gorge" (as in "autumn"), or "gowge" (as in "Audi"); other examples are "ou", "ough" (with 8 different pronunciations);

- letters which do not exist in L1 e.g. " $v$ " pronounced " $w$ " by speakers of South Asian languages - and the reverse for Germanic languages.

All these can have an impact on a student's achievement in an oral reading test. Additionally, both listening and speaking have an impact on a student's ability to write good English, but new arrivals are often placed in the lowest ability sets or withdrawn from the class for small group or individual teaching so they miss the opportunity for valuable interaction with peer role models.

\subsection{Reading}

Decoding is often taught by using phonics, the sound of letters rather than their names. Although most English students will have learned these sounds early in their school lives, new arrivals have to learn these whilst also being taught subject content. As seen above, many English words do not follow phonic rules. In the early stages of reading (BICs) [7], it is very common to hear students reading a book fluently without comprehension. As seen in 4.2, many words in English have several meanings, for example "habit" meaning something done regularly as well as the 
garment worn by a monk. It can also be in a word like "inhabit" and "habitat" connected with places to live. This also applies in Science vocabulary such as "force, tissue, cell" [8], which have different meanings in rigorous scientific language, compared with colloquial usage in the wider world. In all cases context is very important for finding the relevant meaning.

In any lesson, teachers may emphasise the key words which are the technical vocabulary to be learned in the lesson. However, they assume that students will also know subject-specific vocabulary as well as the syntax (word order) and grammar in the English carrying language, which can be used to elicit the meaning of unknown words. Inference and deduction are higher level reading skills requiring CALP [7] where links are made with prior knowledge to work out meanings. Most written texts, including reading assessments, involve an awareness of context, culture and heritage, such as phrases from the Bible and Shakespeare, which is often outside the experience of EAL students from totally different cultures. Idiomatic language such as imagery, metaphors and similes is used regularly to imply deeper meanings but it makes no sense when translated literally. This has an implication in assessments where one unknown or misinterpreted word can be a barrier to higher achievement - for example, misunderstanding the term "divine intervention" meaning "miracle" meant 0 marks for one A Level RE student in the UK. Students who are literate in their L1 are often encouraged to read English texts being studied (such as novels) in their L1 so that they can gain comprehension and be able to discuss in a group or whole class situation.

\subsection{Writing}

At word level, difficulties can occur with writing down letters because the orientation in some languages is different from English, for example, Arabic, Hebrew and Urdu are written right to left; Japanese and Chinese may be written from top to bottom. These languages and others such as Russian and Greek have totally different scripts from English. Lower case and upper case rules are different in English - some languages use capital letters for all nouns, whereas others use only lower case.

Spelling mistakes are common for several reasons: English is not a perfectly phonetic language (see 4.2 above); it has spelling rules but there are many words which break the rules; homophones and homonyms such as there, their and they're, to, too, two cause confusion; the wrong word family might be used such as "practise" (verb) instead of "practice" (noun); or the wrong noun e.g. "adultery" instead of "adulthood". "Led" and "lead" (the metal) are particular challenges, even for native English speakers.

Vocabulary is a problem, as discussed in 4.2 and 4.3. Both paper-based and computer-aided dictionaries become problematic when multiple meanings are given, whilst a thesaurus used to vary or improve vocabulary often results in the use of words which are inappropriate for the context. Singular nouns that stand for a plural idea are confusing, such as "suit" or "pack of cards", "staff", "team", "pair", "both". Uncountable words such as "some, much, many, few, more, several" and modal verbs such as "could, would and should" are also difficult to use.

At sentence level, syntax can cause problems because word order can be very different in other languages. English grammar is difficult for EAL learners: verb tenses are often mixed inappropriately and past tense is particularly difficult because English has many irregular verbs e.g. "take" becomes "took", so logically students might use "tooken" instead of "was taken" or following regular past tense rules, e.g. "buyed" instead of "bought" (particularly in mathematics questions). Errors are made in subject/verb agreement - plural verb with a singular subject and vice-versa; passive voice using the verb "to be" - "is, are, am, was, were"; pronouns such as "he" and "she" because of unfamiliar names or because some languages have masculine and feminine words; third person "it" - particularly when using the passive voice; inappropriate use of prepositions and comparatives and superlatives - "more better" or "gooder", "most worst" or "worser". A regular difficulty is the confusion in the use of definite and indefinite articles "the", "a" and "an". Students may also have a limited range of punctuation or forget to use any. There could be 
different symbols to use in other languages and speech punctuation and apostrophes are particularly problematic.

At whole text level students have to learn about form and function:

- different genres (such as persuasion, advice, instructions, explanations, descriptions, comparisons) require different ingredients (sentence starters, word order, verb tenses, vocabulary, connectives...) because they have different purposes.

- writing comes in different forms: instructions, recipes, letters, plays, poems, advertisements, novels and

- it has to be tailored for different audiences which require formal or informal vocabulary.

There is a lot to learn in order to secure cognitive academic language proficiency (CALP) [7].

\section{The English needed in assessments}

Academic language has a crucial role in educational achievement but in many cases students learning EAL have no experience of academic language in their L1 at home to draw on. The acquisition of any language comes from teaching, reading, experiences and perceptions of the language. Assessments then test how much of the input has been learned so that progress can be recorded. Assessments in any subject require the student to read and understand what the question says, then write a logical response which answers the question. However, assessment questions never ask a student to write down everything they know about a subject. The questions ask for specific information to test whether a student has really understood the concepts but the wording in the instructions can be challenging for a learner of EAL, for example:

- Various words are used instead of saying "write" for example "Give...", "State...", "Say...", "Comment..." and "In what way..."

- "Explain how..." - implies the way in which something happens, whereas "explain why" expects a reason or cause.

- Instructions such as "Discuss...", "Compare...", "Contrast..." or "Suggest..." expect a student to be able to give opinions sometimes on both sides of an argument and make a conclusion.

- Commands such as: “Analyse...", “Evaluate...", "Synthesise...", “Predict...", “How could..." all require CALP [7] in the format appropriate for the genre such as verb tenses, modal verbs, connectives and sentence starters to answer them.

- Some more complicated instructions such as: "Give two significant features...", "Give one benefit... or drawback...", "Give an advantage/disadvantage/benefit/drawback...".

- Phrases expecting quotations from the text have to be taught too: "Find a phrase which gives a negative/positive impression...", "How does the writer's use of language..." "Illustrate your answer with...", "Give evidence that...".

As a concession, EAL students who have been in the UK less than two years are allowed the use of a paper-based bilingual dictionary, not any electronic device. They are also allowed $25 \%$ extra time because of the extra time taken to use the dictionary.

\section{Possible benefits of computer-aided translation tools}

Sections 4 and 5 have detailed many of the subtle linguistic problems EAL learners have in acquiring the academic language proficiency required to reach their full academic potential in assessments at all levels. So are mainstream teachers justified in using computer-aided translation tools to provide access to the curriculum for their students? In order to answer this question, the author discussed the use of e-translation tools on-line with experienced colleagues in all sectors of education $[9,10,11]$ and referred to an action research project by NALDIC and BECTA in 2005-6 [12].

There was a general consensus that all the e-translation tools accessed were useful for translating individual words and phrases. For example, Babelfish [13] (with 14 languages) allowed a Dutch speaking student to both access the curriculum and show her understanding in case study 1 
(CS1) [12]; Turkish boys used InterTran (only in Turkish) [14] and particularly liked the 'drop down box' [12] (CS4) as they were able to discuss the alternative choices to gain meaning; Poltran [15] (only in Polish) used pictures as well as the dictionary to support learning [12] (CS2) and Tenar [16] in Polish (CS6) successfully used photographs to illustrate words with multiple meanings. Kumar [10] reported that Mantralingua's Talking Pens [17] and several other online resources were useful in the initial stages of language development as they included images and spoken words to aid understanding. Talking Pens also have the ability to record words spoken by the teacher or child in context. Case study 7 [12] suggested that Survol [18] was quicker to translate Maltese than a paper-based dictionary. Survol and Sakhr [19] (CS 5 translating Arabic) were especially useful with older and more able EAL students who could use the internet to provide instant access to photographs and images to gain understanding.

Pim [9] explained another important advantage of e-translation tools because they acknowledge the first language of the individual student and thus raise self esteem and confidence. In CS1 [12] a Dutch student was able to access lessons alongside other pupils as well as to demonstrate her understanding enough to participate in conversation rather than feeling isolated as the only Dutch speaker in the class. Students in the case studies were generally happy to use e-translation tools as they often led to more independence when they usually experiment with translations of other works while online.

\section{Some of the problems and drawbacks with such tools, that create barriers to acquiring full academic fluency and thus pose challenges to future translation tool developers.}

The most common problem encountered by experienced colleagues $[9,10,11]$ and in the action research $[12,20]$ was that the e-translations used "were not sufficiently advanced or reliable... beyond isolated word level" [12]. Luukko-Vinchenzo [11] agreed that in ESOL lessons they were unreliable for complex sentences with several clauses and at whole-text level where it is essential that the student is aware of the context, genre, purpose and form. Brentnall [9] explained they do not work perfectly, provide poor and often inaccurate language and therefore are not the most effective teaching tool. CS1 and 4 had to re-work more complicated sentences into simple statements to gain understanding, CS 1, 4 and 5 referred to translating back from L1 to check for accuracy. Jarrett [9] spoke of using a piece of translated text and editing errors so that gaps in knowledge could be identified by the teacher.

It is clear that most translation tools require intensive support from an adult with a good command of English to aid translation, break instructions down, explain carefully and re-work poor translations, which is very time-consuming: most teachers have to prepare translation lists before the lesson.

There are no e-translation tools for some languages and some tools are for only one language e.g. Poltran and Sakhr, yet as seen in section 1, there are more than 300 languages used in the UK alone. Additionally, several case studies reported that it is not always easy to be sure that the translation tool gave the right meaning for the context where synonyms and homonyms are used: for example, in Science lessons (CS2 and CS3) where Urdu translation tools [21,22] gave different translations for the same word. Technical words in any subject are particularly difficult to translate (CS2).

Several studies record problems with the speed of e-translation tools when Internet traffic is heavy. This can have adverse effects on motivation and behaviour. Others report that the e-tools are time consuming, for example, CS4 found that problems in translating instructions, modal verbs like "could" and "might", carrying words like "got", "some" and "did", in exam questions, took so much time that only 3 out of 20 questions had been answered in the lesson. For poetry in GCSE English assessments in the UK, translation tools would not be able to help an EAL student understand challenging specific vocabulary such as idioms, metaphors, similes and imagery to convey meaning through inference (CS7). As a result some teachers abandon the e-translation tools and find another method. 
Another problem is that e-translation tools are often used in sessions where students are withdrawn from the mainstream classroom. This can isolate new arrivals as they are not hearing good examples of the target language being spoken or developing peer to peer social skills in group and whole class situations.

Ideally L1 and L2 should develop alongside each other but some students are not literate in their L1 as the language spoken at home is more social. In CS3 the EAL students spoke Mirpuri rather than Urdu. On the other hand the Polish student in CS2 reported that he was forgetting his home language as his parents insisted that he should use English at home.

It is not always possible to access computers in every classroom and it can be distracting to the other pupils and teacher when they are used for individual work in a mainstream classroom. Finally, it may not be possible for some EAL students to make use of e-tools for homework as they may not have a computer or Internet access at home.

\section{Potential Effects of Emerging and Future Technologies}

E-translation has come a long way in recent decades. Even in the late 20th century it was still the stuff of science fiction and yet now it is accepted as an established technology, even with its many flaws. In parallel there has been the development of direct dictation and speech recognition, which have also evolved from science fiction to widespread usage over a quarter of a century. These evolutionary developments may be very largely ascribed to the effect of Moore's Law [23] and, given the general view that the progression envisaged by Moore will continue into the foreseeable future, it is appropriate to consider how this will affect the evolution of e-translation tools.

The march of Moore's law has been extrapolated by some, e.g. Kurzweil [24], to postulate a situation where computers become more intelligent than human beings, with some predictions seeing this as early as the middle of the present century. In many specialized areas, computers are already far more able than human beings, but these areas are only where they can be finely programmed to excel at a single task. For anything approaching human reasoning ability to be developed, a computer would need to absorb massive learning about context.

The problem of e-translation illustrates the need for contextual understanding quite clearly, since meanings of words, entire texts and especially idiomatic constructs, are deeply linked to context as absorbed by the human being. A child in secondary school, for example, will have had at least 10 years of rich context absorption from the wide-ranging experiences of "real-life" and the importing of such experience into a computer is still in the realm of science fiction: the nearest analogy at present is probably the way in which algorithms harvest knowledge of human proclivities from social media.

There is an interesting analogy with the concept of the "uncanny valley", which has become a talking point in the fields of computer graphics and chatbots $[25,26]$. This is based on the observation that advances in simulating a human being either visually (as in computer graphics) or orally (as in chatbots) have produced impressive achievements, but when they become relatively close to the behaviour of a real human being, there is an undefinable unnerving nature to them that still distances them from the perceived behaviour of a real human being, hence they are "uncanny". It is suggested that there will be an analogy in e-translation, where translations will evolve to become extremely good, but yet still be identifiable as not being the work of a real human being: this is a topic that merits some further study.

\section{Conclusions: Challenges for future translation tool developers.}

It is clear that machines are not likely to fully replace human translators in the near future. Kelly [27] believes that there are six reasons for this:

(i) It's tough to get good translation even from perfectly bilingual human beings

(ii) Translation quality is highly subjective

(iii) There are too many languages out there

(iv) Most languages are not written 
(v) Context is key

(vi) Language is just too important

Together, these can be classed as "subtleties" in language that represent intractable challenges for current algorithms. The majority of leading developers of machine translation are experts in mathematics but have little knowledge of linguistics, foreign languages or literature [2]. Although there seems to be an acceptance that the method based on the expertise of language specialists would be better, computers are not yet powerful enough to implement it. This necessarily raises the question of the influence of Moore's Law, which has driven the increase of computer power for several decades, and is predicted to continue to do so for at least a few more. This suggests that computer (and Internet) power will provide translation services of steadily increasing quality, but the discussion presented above suggests that something approaching perfection would require a substantially different architecture, in particular one that is able to garner far more information about context, both physical and cultural, as well as other aspects (e.g. whether the piece is poetry). These are immense but intriguing challenges for the computing and software communities, but the reasoning presented here shows that it is essential that they work in close collaboration with a spectrum of experienced practitioners in the fields of traditional translation and multilingual education.

\section{Note}

This paper is an edited and expanded version of one originally presented at the International Conference on Recent Advances in Computer Systems, University of Haíl, Haíl, Kingdom of Saudi Arabia in 2015 [28].

\section{References}

[1] UK Government, “Languages Spoken by Pupils in England". London: National Level Data, January 2015.

[2] Lewis-Kraus, G. "Is Translation an Art or a Maths Problem?" In The New York Times magazine: The Tech and Design Issue, New York, USA. June 4th 2015. Available: http://www.nytimes.com/2015/06/07/ magazine/is-translation-an-art-or-a-math-problem.html? $\mathrm{r}=0$.

[3] Sidorov, G. "Syntactic Dependency Based N-grams in Rule Based Automatic English as Second Language Grammar Correction", International Journal of Computational Linguistics and Applications, vol. 4, no. 2, pp. 169-188, 2013. Available: http://www.cic.ipn.mx/ sidorov/IJCLA_SN_GRAMS 2013.pdf

[4] Monroe, D. "Deep Learning Takes on Translation”, Communications of the ACM, Vol. 60, No.6, pp12-14, 2017.

[5] Dorren, G., quoted in “Will English Continue to Reign as the world's Most Common Language?" Qrius (digital magazine) 08:02:19. Ahmedabad, India: Perfect Creative Media Pvt. Ltd.

[6] Kapitanov, A., et al, Clustering of Word Contexts as a Method of Eliminating Polysemy of Words", IEEE Conf. of Russian Young Researchers in Electrical and Electronic Engineering, 28-31 Jan 2019. Nat. Res. Univ. for Electronic Tech. MIET, Zelenograd, Moscow. Paper 158, 4pp.

[7] Cummins, J. "Bilingualism and special education", Clevedon: Multilingual Matters, 1984.

[8] UK Government, Department for Education and Science (DfES), “Key Stage 3 National Strategy. Access and Engagement in Science: Teaching pupils for whom English is an additional language", London: DfES, 2002.

[9] Jarrett, M., Brentnall, J., Pim, C. “Translation Apps": discussion 3758 (11th February 2015). In: eal-bilingual@googlegroups.com.

[10] Kumar, R. Excell, D. Use of computer-aided translation tools for Early Years and Primary pupils, Bradford, UK: e-conversation, 2015. 
[11] Luukko-Vinchenzo, L. Excell, D. Use of computer aided translation tools for ESOL adult learners, Wrexham, UK: e-conversation, 2015.

[12] NALDIC with BECTA. "Case Studies: action research project on e-translation in the classroom", Coventry, UK 2007. Available: http://www.naldic.org.uk/eal-teaching-and-learning/eal-resources/ict.

[13] BabelFish - Free Online Translator. Accessed 4 March 2019. https://babelfish.com.

[14] Translation Experts Ltd, InterTran Web Site Translation Server. Accessed 4 March 2019: http://www.tranexp.com:2000/InterTran/InterTran.

[15] Ectaco Inc., "Poltran.com Stream Translation Technologies”, Accessed 4 March 2019: www.poltran.com.

[16] Tenar Polish translation tool. Now not online; formerly: www.tenar.tpi.pl/ Referenced in "Case Study 6: Using e-translation with newly arrived Polish pupils", NALDIC with BECTA, op. cit., 2007. Available at: http://www.naldic.org.uk/Resources/NALDIC/Teaching\%20and\%20Learning/Documents/CaseStudy6. pdf.

[17] NALDIC EAL and ICT Vodcast - Programme 6 Mantralingua's Recordable Talking Pen, Available: http://www.naldic.org.uk/eal-teaching-and-learning/eal-resources/ict.

[18] Survol Maltese translation tool. Now not online; formerly: http://www.survol.com/hierflier/e2mdemo/. Referenced in "Case Study 7: Using a translation website to support a Maltese Year 11 pupil”, NALDIC with BECTA, op. cit., 2007. Available at: https://www.naldic.org.uk/Resources/NALDIC/Teaching\%20 and\%20Learning/Documents/casestudy7.pdf.

[19] Sakhr Arabic translation tool. Now not online; formerly: http://translate.sakhr.com/sakhr/elogin ET.asp Referenced in World Association of Arab Translators and Linguists website, accessed 4 March 2019.

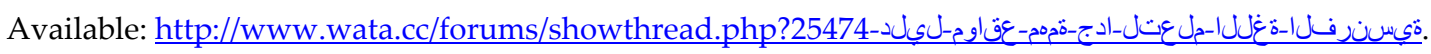

[20] Davies, N. and Lama, D. “It's too slow. It doesn't make sense. I'll ask my friend to help me - it's better!", in "Using e-translation in the classroom". NALDIC Quarterly 4.2. Luton: NALDIC, 2007.

[21] Platts, J.T., “A dictionary of Urdu, classical Hindi, and English”. London: W. H. Allen \& Co., 1884. Available online at: http://dsal.uchicago.edu/dictionaries/platts.

[22] Siddiqui, W. "English-Urdu Dictionary", 2005. Available online at: http://biphost.spray.se/tracker/dict/

[23] Moore, G.E., “Cramming More Components onto Integrated Circuits,” Electronics, pp. 114-117, April 19, 1965.

[24] Kurzweil, R., “The Singularity is Near”, New York, Viking, 2005.

[25] Mori, M., “On the Uncanny Valley.” Proc. Humanoids 2005 Workshop: Views of the Uncanny Valley, Tsukuba, Japan, December 2005.

[26] Tunkelang, D., “The Uncanny Valley of Chatbots", Medium Community blog posting, Sep 6, 2016. Available: https://medium.com/@dtunkelang/the-uncanny-valley-of-chatbots-9f265b42d83b.

[27] Kelly, N. (2014). "Why machines alone cannot solve the world's translation problem”, Huffington Post 1.9.2014. Available: http://www.huffingtonpost.com/nataly-kelly/why-machines-alone-cannot-translatio n b 4570018.html.

[28] Excell, D., “Subtleties: Some Challenges in Achieving Second Language Fluency using Computer-Aided Translation Tools in Education", in the Proceedings of the 2015 International Conference on Recent Advances in Computer Systems, 30th November - 31st December 2015, University of Haíl, Haíl, KSA, DOI: 10.2991/racs-15.2016.18. Available: https://www.atlantis-press.com/proceedings/racs-15/25847791. 